\title{
Bounds for Eigenvalues of Arrowhead Matrices and Their Applications to Hub Matrices and Wireless Communications
}

\author{
Lixin Shen ${ }^{1}$ and Bruce W. Suter ${ }^{2}$ \\ ${ }^{1}$ Department of Mathematics, Syracuse University, Syracuse, NY 13244, USA \\ 2 Air Force Research Laboratory, RITC, Rome, NY 13441-4505, USA \\ Correspondence should be addressed to Bruce W. Suter, bruce.suter@rl.af.mil
}

Received 29 June 2009; Accepted 15 September 2009

Recommended by Enrico Capobianco

This paper considers the lower and upper bounds of eigenvalues of arrow-head matrices. We propose a parameterized decomposition of an arrowhead matrix which is a sum of a diagonal matrix and a special kind of arrowhead matrix whose eigenvalues can be computed explicitly. The eigenvalues of the arrowhead matrix are then estimated in terms of eigenvalues of the diagonal matrix and the special arrowhead matrix by using Weyl's theorem. Improved bounds of the eigenvalues are obtained by choosing a decomposition of the arrowhead matrix which can provide best bounds. Some applications of these results to hub matrices and wireless communications are discussed.

Copyright ( $) 2009$ L. Shen and B. W. Suter. This is an open access article distributed under the Creative Commons Attribution License, which permits unrestricted use, distribution, and reproduction in any medium, provided the original work is properly cited.

\section{Introduction}

In this paper we develop lower and upper bounds for arrowhead matrices. A matrix $Q \in \mathbb{R}^{m \times m}$ is called an arrowhead matrix if it has a form as follows:

$$
Q=\left[\begin{array}{ll}
D & c \\
c^{t} & b
\end{array}\right],
$$

where $D \in \mathbb{R}^{(m-1) \times(m-1)}$ is a diagonal matrix, $c$ is a vector in $\mathbb{R}^{m-1}$, and $b$ is a real number. Here the superscript " $t$ " signifies the transpose. The arrowhead matrix $Q$ is obtained by bordering the diagonal matrix $D$ by the vector $c$ and the real number $b$. Hence, sometimes the matrix $Q$ in (1) is also called a symmetric bordered diagonal matrix. In physics, arrowhead matrices have been used to describe radiationless transitions in isolated molecules [1] and oscillators vibrationally coupled with a Fermi liquid [2]. Numerically efficient algorithms for computing eigenvalues and eigenvectors of arrowhead matrices were discussed in [3]. The properties of eigenvectors of arrowhead matrices were studied in [4], and as an application of their results, an alternative proof of Cauchy's interlacing theorem was given there. The existence of arrowhead matrices was investigated recently in [5-8] such that the constructed arrowhead matrix has the pregiven eigenvalues and other additional requirements.

Our motivation to study lower and upper bounds of arrowhead matrices is from Kung and Suter's recent work on the hub matrix theory [9] and its applications to multipleinput and multiple output (MIMO) wireless communication systems. A matrix, say $A$, is a hub matrix with $m$ columns if its first $m-1$ columns (called nonhub columns) are orthogonal to each other with respect to the Euclidean inner product and its last column (called hub column) has a Euclidean norm greater than any other columns. Subsequently, it was shown that the Gram matrix of $A$, that is, $Q=A^{t} A$, is an arrowhead matrix and its eigenvalues could be bounded by the norms of the columns of $A$. As pointed out in [9-11], the eigenstructure of $Q$ determines the properties of wireless communication systems. This motivates us to reexamines these bounds of the eigenvalues of $Q$ and makes them sharper. In [9], the hub matrix theory is also applied to the MIMO beamforming problem by comparing $k$ of $m$ transmitting antennas with the largest signal-to-noise ratio, including the special case where $k=1$ which corresponds to a transmitting hub. The relative performance of resulting system can be expressed as the ratio of the largest eigenvalue 
of the truncated $Q$ matrix to the largest eigenvalue of the $Q$ matrix. Again, it was previously shown that these ratios could be bounded by the ratios of norms of columns of the associated hub matrix. Sharper bounds will be presented in Section 4.

The well-known result on the eigenvalues of arrowhead matrices is the Cauchy interlacing theorem for Hermitian matrices [12]. We assume that the diagonal elements $d_{j}$, $j=1,2, \ldots, m-1$, of the diagonal matrix $D$ in (1) satisfy the relation $d_{1} \leq d_{2} \leq \cdots \leq d_{m-1}$. Let $\lambda_{1}, \lambda_{2}, \ldots, \lambda_{m}$ be the eigenvalues of $Q$ arranged in increasing order. The Cauchy interlacing theorem says that

$$
\lambda_{1} \leq d_{1} \leq \lambda_{2} \leq d_{2} \leq \cdots \leq d_{m-2} \leq \lambda_{m-1} \leq d_{m-1} \leq \lambda_{m} .
$$

When the vector $c$ and the real number $b$ in (1) are taken into consideration, a lower bound of $\lambda_{1}$ and an upper bound of $\lambda_{m}$ were developed by using the well-known Gershgorin theorem (see, e.g., $[3,12]$ ), that is,

$$
\begin{aligned}
& \lambda_{m}<\max \left\{d_{1}+\left|c_{1}\right|, \ldots, d_{m-1}+\left|c_{m-1}\right|, b+\sum_{i=1}^{m-1}\left|c_{i}\right|\right\} \\
& \lambda_{1}>\min \left\{d_{1}-\left|c_{1}\right|, \ldots, d_{m-1}-\left|c_{m-1}\right|, b-\sum_{i=1}^{m-1}\left|c_{i}\right|\right\} .
\end{aligned}
$$

Accurate bounds of eigenvalues of arrowhead matrices are of great interest in applications as mentioned before. The main results of this paper are presented in Theorems 11 and 12 for the upper and lower bounds of the arrowhead matrices. It is also shown in Corollary 13 that the resulting bounds are tighter than in (2), (3), and (4).

The rest of the paper is outlined as follows. In Section 2, we will introduce notation and present several useful results on the eigenvalues of arrowhead matrices. We give our main results in Section 3. In Section 4, we revisit the lower and upper bounds of the ratio of eigenvalues of arrowhead matrices associated with hub matrices and wireless communication systems [9], and subsequently, we make those bounds shaper by using the results in Section 3. In Section 5, we compute the bounds of arrowhead matrices using the developed theorems via three examples. Conclusions are given in Section 6.

\section{Notation and Basic Results}

The identity matrix is denoted by $I$. The notation $\operatorname{diag}\left(a_{1}, a_{2}, \ldots, a_{n}\right)$ represents a diagonal matrix whose diagonal elements are $a_{1}, a_{2}, \ldots, a_{n}$. The determinant of a matrix $A$ is denoted by $\operatorname{det}(A)$. The eigenvalues of a symmetric matrix $A \in \mathbb{R}^{n \times n}$ are always ordered such that

$$
\lambda_{1}(A) \leq \lambda_{2}(A) \leq \cdots \leq \lambda_{n}(A)
$$

For a vector $a \in \mathbb{R}^{n}$, its Euclidean norm is defined to be $\|a\|:=\sqrt{\sum_{i=1}^{n}\left|a_{i}\right|^{2}}$.

The first result is about the determinant of an arrowhead matrix and is stated as follows.
Lemma 1. Let $Q \in \mathbb{R}^{m \times m}$ be an arrowhead matrix of the form (1), where $D=\operatorname{diag}\left(d_{1}, d_{2}, \ldots, d_{m-1}\right) \in \mathbb{R}^{(m-1) \times(m-1)}, b \in \mathbb{R}$, and $c=\left(c_{1}, c_{2}, \ldots, c_{m-1}\right) \in \mathbb{R}^{m-1}$. Then

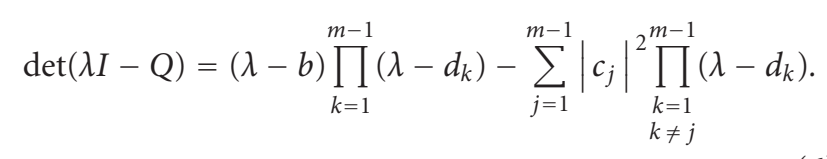

The proof of this result can be found in $[5,13]$ and therefore is omitted here.

When the diagonal matrix $D$ in (1) is a zero matrix, the following result is followed from Lemma 1.

Corollary 2. Let $Q \in \mathbb{R}^{m \times m}$ be an arrowhead matrix having the following form:

$$
Q=\left[\begin{array}{ll}
0 & c \\
c^{t} & b
\end{array}\right],
$$

where $c$ is a vector in $\mathbb{R}^{m-1}$ and $b$ is a real number. Then the eigenvalues of $Q$ are

$$
\begin{gathered}
\lambda_{1}(Q)=\frac{b-\sqrt{b^{2}+4\|c\|^{2}}}{2}, \quad \lambda_{m}(Q)=\frac{b+\sqrt{b^{2}+4\|c\|^{2}}}{2}, \\
\lambda_{i}(Q)=0, \quad \text { for } i=2, \ldots, m-1 .
\end{gathered}
$$

Proof. By using Lemma 1, we have

$$
\operatorname{det}(\lambda I-Q)=\lambda^{m-2}\left(\lambda^{2}-b \lambda-\|c\|^{2}\right)
$$

Clearly, $\lambda=0$ is a zero of $\operatorname{det}(\lambda I-Q)$ with multiplicity $m-2$. The zeros of the quadratic polynomial $\lambda^{2}-b \lambda-\|c\|^{2}$ are $\left(b-\sqrt{b^{2}+4\|c\|^{2}}\right) / 2$ and $\left(b+\sqrt{b^{2}+4\|c\|^{2}}\right) / 2$, respectively. This completes the proof.

In what follows, a matrix $Q$ having a form in (7) is called a special arrowhead matrix. The following corollary (also, see [3]) is a direct result from Lemma 1.

Corollary 3. Let $Q$ be an $m \times m$ arrowhead matrix given by (1), where $D=\operatorname{diag}\left(d_{1}, d_{2}, \ldots, d_{m-1}\right) \in \mathbb{R}^{(m-1) \times(m-1)}, b \in \mathbb{R}$, and $c=\left(c_{1}, c_{2}, \ldots, c_{m-1}\right) \in \mathbb{R}^{m-1}$. Let us denote the repetition of the number $d_{j}$ in the sequence $\left\{d_{i}\right\}_{i=1}^{m-1}$ by $k_{j}$. If $k_{j} \geq 2$, then $d_{j}$ is the eigenvalue of $Q$ with multiplicity $k_{j}-1$.

Proof. When the integer $k_{j} \geq 2$, the result follows from Lemma 1 since $\left(\lambda-d_{j}\right)^{k_{j}-1}$ is a factor of the polynomial $\operatorname{det}(\lambda I-Q)$.

Corollary 4. Let $Q$ be an $m \times m$ arrowhead matrix given by $(1)$, where $D=\operatorname{diag}\left(d_{1}, d_{2}, \ldots, d_{m-1}\right) \in \mathbb{R}^{(m-1) \times(m-1)}$, $b \in \mathbb{R}$, and $c=\left(c_{1}, c_{2}, \ldots, c_{m-1}\right) \in \mathbb{R}^{m-1}$. Suppose that the last $k \geq 2$ diagonal elements $d_{m-k}, d_{m-k+1}, \ldots, d_{m-1}$ of $D$ are 
identical and distinct from the first $m-k-1$ diagonal elements $d_{1}, d_{2}, \ldots, d_{m-k-1}$ of $D$. Define a new matrix

$$
\widetilde{Q}:=\left[\begin{array}{ccccc}
d_{1} & & & & \tilde{c}_{1} \\
& \ddots & & & \vdots \\
& & d_{m-k-1} & & \tilde{c}_{m-k-1} \\
& & & d_{m-k} & \tilde{c}_{m-k} \\
\tilde{c}_{1} & \cdots & \tilde{c}_{m-k-1} & \tilde{c}_{m-k} & b
\end{array}\right]
$$

with $\tilde{c}_{j}=c_{j}$ for $j=1,2, \ldots, m-k-1$ and $\tilde{c}_{m-k}=$ $\sqrt{\sum_{j=m-k}^{m-1}\left|c_{j}\right|^{2}}$. Then the eigenvalues of $Q$ are that of $\tilde{Q}$ together with $d_{m-k}$ with multiplicity $k-1$.

Proof. Since numbers $d_{m-k}, d_{m-k+1}, \ldots, d_{m-1}$ are identical and distinct from numbers $d_{1}, d_{2}, \ldots, d_{m-k-1}$, we have

$$
\begin{gathered}
\prod_{\substack{i=1 \\
i \neq j}}^{m-1}\left(\lambda-d_{i}\right)=\left(\prod_{\substack{i=1 \\
i \neq j}}^{m-k}\left(\lambda-d_{i}\right)\right)\left(\lambda-d_{m-k}\right)^{k-1}, \quad j \leq m-k-1, \\
\sum_{j=m-k}^{m-1}\left|c_{j}\right|^{2} \prod_{\substack{i=1 \\
i \neq j}}^{m-1}\left(\lambda-d_{i}\right) \\
=\left(\sum_{j=m-k}^{m-1}\left|c_{j}\right|^{2}\right)\left(\prod_{i=1}^{m-k-1}\left(\lambda-d_{i}\right)\right)\left(\lambda-d_{m-k}\right)^{k-1} .
\end{gathered}
$$

By (6) in Lemma 1, we have

$$
\begin{aligned}
& \operatorname{det}(\lambda I-Q) \\
& =(\lambda-b) \prod_{i=1}^{m-1}\left(\lambda-d_{i}\right)-\sum_{j=1}^{m-1}\left|c_{j}\right|^{2} \prod_{\substack{i=1 \\
i \neq j}}^{m-1}\left(\lambda-d_{i}\right) \\
& =(\lambda-b) \prod_{i=1}^{m-k}\left(\lambda-d_{i}\right)-\sum_{j=1}^{m-k}\left|\tilde{c}_{j}\right|^{2} \prod_{\substack{i=1 \\
i \neq j}}^{m-k-1}\left(\lambda-d_{i}\right) \\
& \left(\lambda-d_{m-k}\right)^{k-1} \\
& =\operatorname{det}(\lambda I-\widetilde{Q}) \cdot\left(\lambda-d_{m-k}\right)^{k-1} .
\end{aligned}
$$

Clearly, if $\lambda$ is an eigenvalue of $Q$, then $\lambda$ is either an eigenvalue of $\widetilde{Q}$ or $d_{m-k}$. Conversely, $d_{m-k}$ is an eigenvalue of $Q$ with multiplicity $k-1$ and the eigenvalues of $\widetilde{Q}$ are that of $Q$. This completes the proof.

By using Corollaries 3 and 4 , to study the eigenvalues of $Q$, we may assume that the diagonal elements $d_{1}, d_{2}, \ldots, d_{m-1}$ of $Q$ are distinct when we study the eigenvalues of $Q$ in (1). Since eigenvalues of square matrices are invariant under similarity transformations, we can without loss of generality arrange the diagonal elements to be ordered so that $d_{1}<$ $d_{2}<\cdots<d_{m-1}$. Furthermore, we may assume that all entries of the vector $c$ in (1) are nonzero. The reason for this assumption is the following. Suppose that $c_{j}$, the $j$ th entry of $c$, is nonzero, it can be easily seen from Lemma 1 that $\lambda-d_{j}$ is a factor of $\operatorname{det}(\lambda I-Q)$; that is, $d_{j}$ is one of eigenvalues of $Q$. The remaining eigenvalues of $Q$ are the same as those of a matrix which is obtained by simply deleting the $j$ th row and column of $Q$. In summary, for any arrowhead matrix, we can find eigenvalues corresponding to repeated values in $D$ or associated with zero elements in $c$ by inspection.

In this paper, we call a matrix $Q$ in (1) irreducible if the diagonal elements $d_{1}, d_{2}, \ldots, d_{m-1}$ of $Q$ are distinct and all elements of $c$ are nonzero. By using Corollary 4 and the above discussion, this arrowhead matrix can be reduced to an irreducible one.

Remark 5. In [4, 9], Hermitian arrowhead matrices are considered; that is, it allows that $c$ in the matrix $Q$ of the form (1) is a vector in $\mathbb{C}^{m-1}$. We can directly construct many (real symmetric) arrowhead matrices denoted by $\widetilde{Q}$ from $Q$. The diagonal elements of these symmetric arrowhead matrices are the exactly same as those of $Q$. The vector $\tilde{c}$ in $\widetilde{Q}$ could be chosen as

$$
\tilde{c}=\left( \pm\left|c_{1}\right|, \pm\left|c_{2}\right|, \ldots, \pm\left|c_{m-1}\right|\right) .
$$

In such a way, there are $2^{m-1}$ such symmetric arrowhead matrices. Because $\operatorname{det}(\lambda I-Q)=\operatorname{det}(\lambda I-\widetilde{Q})$ by Lemma 1, every such symmetric arrowhead matrix $\widetilde{Q}$ has the identical eigenvalues with $Q$. This is the reason why we just consider the eigenvalues of real arrowhead matrices in this paper.

The following well-known result by Weyl on eigenvalues of a sum of two symmetric matrices is used in the proof of our main theorem.

Theorem 6 (Weyl). Let $F$ and $G$ be two $m \times m$ symmetric matrices. Let us assume that the eigenvalues of $F, G$, and $F+G$ have been arranged in increasing order. Then

$$
\begin{array}{ll}
\lambda_{j}(F+G) \leq \lambda_{i}(F)+\lambda_{j-i+m}(G), & \text { for } i \geq j, \\
\lambda_{j}(F+G) \geq \lambda_{i}(F)+\lambda_{j-i+1}(G), & \text { for } i \leq j .
\end{array}
$$

Proof. See [14, page 62] or [12, page 184].

To apply Theorem 6 for estimating eigenvalues of an irreducible arrowhead matrix $Q$, we need to decompose $Q$ into a sum of two symmetric matrices whose eigenvalues are relatively easy to be computed. Motivated by the structure of the arrowhead matrix and the eigenstructure of a special arrowhead matrix (see, Corollary 2), we write $Q$ into a sum of a diagonal matrix and a special arrowhead matrix.

To be more precisely, let $Q \in \mathbb{R}^{m \times m}$ be an irreducible arrowhead matrix as follows:

$$
Q=\left[\begin{array}{cc}
D & c \\
c^{t} & d_{m}
\end{array}\right],
$$


where $d_{m} \in \mathbb{R}, D=\operatorname{diag}\left(d_{1}, d_{2}, \ldots, d_{m-1}\right)$ with $0 \leq d_{1}<$ $d_{2}<\cdots<d_{m-1} \leq d_{m}$, and $c$ is a vector in $\mathbb{R}^{m-1}$. For a given $\rho \in[0,1]$, we write

$$
Q=E+S,
$$

where

$$
E=\operatorname{diag}\left(d_{1}, d_{2}, \ldots, d_{m-1}, \rho d_{m}\right), \quad S=\left[\begin{array}{cc}
0 & c \\
c^{t} & (1-\rho) d_{m}
\end{array}\right] .
$$

Therefore, we can use Theorem 6 to give estimates of the eigenvalues of $Q$ via those of $E$ and $S$. To number the eigenvalues of $E$, we introduce the following definition.

Definition 7. For a number $\rho \in[0,1]$, we define an operator $\mathcal{T}_{\rho}$ that maps a sequence $\left\{d_{i}\right\}_{j=1}^{m}$ satisfying $0 \leq d_{1}<d_{2}<$ $\cdots<d_{m-1} \leq d_{m}$ to a new sequence $\left\{\tilde{d}_{i}\right\}_{j=1}^{m}:=\mathcal{T}_{\rho}\left(\left\{d_{i}\right\}_{j=1}^{m}\right)$ according to the following rules: if $\rho d_{m} \leq d_{1}$, then $\tilde{d}_{1}:=\rho d_{m}$ and $\tilde{d}_{j+1}:=d_{j}$ for $j=1, \ldots, m-1$; if $\rho d_{m}>d_{m-1}$, then $\tilde{d}_{j}:=d_{j}$ for $j=1, \ldots, m-1$ and $\tilde{d}_{m}:=\rho d_{m}$; otherwise, there exists an integer $j_{0}$ such that $d_{j_{0}}<\rho d_{m} \leq d_{j_{0}+1}$, then $\tilde{d}_{j}:=d_{j}$ for $j=1, \ldots, j_{0}, \tilde{d}_{j_{0}+1}:=\rho d_{m}$, and $\tilde{d}_{j+1}:=d_{j}$ for $j=j_{0}+1, \ldots, m-1$.

Theorem 8. Let $Q \in \mathbb{R}^{m \times m}$ be an irreducible arrowhead matrix having a form of (16), where $D=$ $\operatorname{diag}\left(d_{1}, d_{2}, \ldots, d_{m-1}\right)$ with $0 \leq d_{1}<d_{2}<\cdots<d_{m-1} \leq d_{m}$, and $c$ is a vector in $\mathbb{R}^{m-1}$. For a given $\rho \in[0,1]$, define $\left\{\tilde{d}_{i}\right\}_{j=1}^{m}:=\mathcal{T}_{\rho}\left(\left\{d_{i}\right\}_{j=1}^{m}\right)$. Then, one has

$$
\begin{aligned}
& \lambda_{j}(Q) \leq \begin{cases}\min \left\{\tilde{d}_{1}+t, \tilde{d}_{2}, \tilde{d}_{m}+s\right\}, & \text { if } j=1, \\
\min \left\{\tilde{d}_{j}+t, \tilde{d}_{j+1}\right\}, & \text { if } 2 \leq j \leq m-1, \\
\tilde{d}_{m}+t, & \text { if } j=m,\end{cases} \\
& \lambda_{j}(Q) \geq \begin{cases}\tilde{d}_{1}+s, & \text { if } j=1, \\
\max \left\{\tilde{d}_{j-1}, \tilde{d}_{j}+s\right\}, & \text { if } 2 \leq j \leq m-1, \\
\max \left\{\tilde{d}_{1}+t, \tilde{d}_{m-1}, \tilde{d}_{m}+s\right\}, & \text { if } j=m,\end{cases}
\end{aligned}
$$

where

$$
\begin{aligned}
& s=\frac{(1-\rho) d_{m}-\sqrt{(1-\rho)^{2} d_{m}^{2}+4\|c\|^{2}}}{2}, \\
& t=\frac{(1-\rho) d_{m}+\sqrt{(1-\rho)^{2} d_{m}^{2}+4\|c\|^{2}}}{2} .
\end{aligned}
$$

Proof. For a given number $\rho \in[0,1]$, we split the matrix $Q$ into a sum of a diagonal matrix $E$ and a special arrowhead matrix $S$ according to (17), where $E$ and $S$ are defined by (18). Clearly, we know that

$$
\lambda_{j}(E)=\tilde{d}_{j}
$$

for $j=1,2, \ldots, m$. By Corollary 2 , we have

$$
\lambda_{1}(S)=s, \quad \lambda_{m}(S)=t, \quad \lambda_{j}(S)=0, \quad \text { for } j=2, \ldots, m-1,
$$

where $s$ and $t$ are given by (21).

Upper Bounds. By (14) in Theorem 6, we have

$$
\lambda_{j}(Q) \leq \lambda_{i}(E)+\lambda_{m+j-i}(S)
$$

for all $i \geq j$. Clearly, for a given $j$,

$$
\lambda_{j}(Q) \leq \min _{i \geq j}\left\{\lambda_{i}(E)+\lambda_{m+j-i}(S)\right\} .
$$

More precisely, since $\left\{\tilde{d}_{i}\right\}_{i=1}^{m}$ is monotonically increasing, $s \leq$ 0 , and $t \geq 0$, we have

$$
\begin{aligned}
\lambda_{1}(Q) & \leq \min \left\{\tilde{d}_{1}+t, \tilde{d}_{2}, \ldots, \tilde{d}_{m-1}, \tilde{d}_{m}+s\right\} \\
& =\min \left\{\tilde{d}_{1}+t, \tilde{d}_{2}, \tilde{d}_{m}+s\right\}, \\
\lambda_{j}(Q) & \leq \min \left\{\tilde{d}_{j}+t, \tilde{d}_{j+1}, \ldots, \tilde{d}_{m}\right\}=\min \left\{\tilde{d}_{j}+t, \tilde{d}_{j+1}\right\}
\end{aligned}
$$

for $j=2, \ldots, m-1$, and

$$
\lambda_{m}(Q) \leq \lambda_{m}(E)+\lambda_{m}(S)=\tilde{d}_{m}+t .
$$

In conclusion, (19) holds.

Lower Bounds. By (15) in Theorem 6, we have, for a given $j$,

$$
\lambda_{j}(Q) \geq \max _{i \leq j}\left\{\lambda_{i}(E)+\lambda_{j-i+1}(S)\right\} .
$$

Hence,

$$
\begin{aligned}
& \lambda_{1}(Q) \geq \lambda_{1}(E)+\lambda_{1}(S)=\tilde{d}_{1}+s, \\
& \lambda_{j}(Q) \geq \max \left\{\tilde{d}_{j}+s, \tilde{d}_{j-1}, \ldots, \tilde{d}_{1}\right\}=\max \left\{\tilde{d}_{j}+s, \tilde{d}_{j-1}\right\}
\end{aligned}
$$

for $j=2, \ldots, m-1$, and

$$
\begin{aligned}
\lambda_{m}(Q) & \geq \max \left\{\tilde{d}_{m}+s, \tilde{d}_{m-1}, \ldots, \tilde{d}_{2}, \tilde{d}_{1}+t\right\} \\
& =\max \left\{\tilde{d}_{m}+s, \tilde{d}_{m-1}, \tilde{d}_{1}+t\right\} .
\end{aligned}
$$

As we can see from Theorem 8, the lower and upper bounds of the eigenvalues for $Q$ are functions of $\rho \in[0,1]$ for the given irreducible matrix $Q$. In other words, the bounds of eigenvalues vary with the number $\rho$. Particularly, when we choose $\rho$ being the ending points, that is, $\rho=0$ and $\rho=1$, we can give an alternative proof of interlacing eigenvalues theorem for arrowhead matrices (see, e.g., [12, page 186]). This theorem is stated as follows. 
Theorem 9 (Interlacing eigenvalues theorem). Let $Q \in \mathbb{R}^{m \times m}$ be an irreducible arrowhead matrix having a form in (16), where $D=\operatorname{diag}\left(d_{1}, d_{2}, \ldots, d_{m-1}\right)$ with $0 \leq d_{1}<d_{2}<\cdots<$ $d_{m-1} \leq d_{m}$, and $c$ is a vector in $\mathbb{R}^{m-1}$. Let the eigenvalues of $Q$ be denoted by $\left\{\lambda_{j}\right\}_{j=1}^{m}$ with $\lambda_{1} \leq \lambda_{2} \leq \cdots \leq \lambda_{m}$. Then

$$
\lambda_{1} \leq d_{1} \leq \lambda_{2} \leq d_{2} \leq \cdots \leq d_{m-2} \leq \lambda_{m-1} \leq d_{m-1} \leq \lambda_{m} .
$$

Proof. By using (19) with $\rho=0$ in Theorem 8 , we have $\lambda_{j} \leq d_{j}$ for $j=1,2, \ldots, m-1$. By using (20) with $\rho=1$ in Theorem 8, we obtain $\lambda_{j} \geq d_{j-1}$ for $j=2,3, \ldots, m$. Combining these two parts together yields our result.

The proof of the above result shows that we could have improved lower and upper bounds for each eigenvalue of an irreducible arrowhead matrix by finding an optimal parameter $\rho$ in $[0,1]$. Our main results will be given in the next section.

\section{Main Results}

Associated with the arrowhead matrix $Q$ in Theorem 8, we define four functions $f_{i}, i=1,2,3,4$, on the interval $[0,1]$ as follow:

$$
\begin{aligned}
& f_{1}(\rho):=\frac{1}{2}\left((1-\rho) d_{m}-\sqrt{(1-\rho)^{2} d_{m}^{2}+4\|c\|^{2}}\right), \\
& f_{2}(\rho):=\frac{1}{2}\left((1-\rho) d_{m}+\sqrt{(1-\rho)^{2} d_{m}^{2}+4\|c\|^{2}}\right), \\
& f_{3}(\rho):=\rho d_{m}+f_{1}(\rho), \\
& f_{4}(\rho):=\rho d_{m}+f_{2}(\rho) .
\end{aligned}
$$

Obviously,

$$
s=f_{1}(\rho), \quad t=f_{2}(\rho),
$$

where $s$ and $t$ are given by (21).

The following observation about monotonicity of functions $f_{i}, i=1,2,3,4$, is simple, but quite useful as we will see in the proof of our main results.

Lemma 10. The functions $f_{1}$ and $f_{2}$ both are decreasing while $f_{3}$ and $f_{4}$ are increasing on the interval $[0,1]$.

The proof of this lemma is omitted.

Theorem 11. Let $Q$ be an irreducible arrowhead matrix defined by (16) and satisfying all assumptions in Theorem 8. Then the eigenvalues of $Q$ are bounded above by

$$
\lambda_{j}(Q) \leq \begin{cases}\min \left\{d_{1}, d_{m-1}+f_{1}\left(\frac{d_{m-1}}{d_{m}}\right)\right\}, & \text { if } j=1, \\ d_{j}, & \text { if } 2 \leq j \leq m-1, \\ d_{m-1}+f_{2}\left(\frac{d_{m-1}}{d_{m}}\right), & \text { if } j=m .\end{cases}
$$

Proof. In Theorem 8, the upper bounds of the eigenvalues of $Q$ in (19) are determined by $\tilde{d}_{j}, j=1,2, \ldots, m$, and $s$ and $t$ in (21). They can be viewed as functions of $\rho$ in $[0,1]$. That is, the upper bounds of the eigenvalues of $Q$ are functions of $\rho$ in the interval $[0,1]$. Therefore, we are able to find optimal bounds of the eigenvalues of $Q$ by choosing proper $\rho$. The upper bounds on $\lambda_{j}(Q)$ for $j=1,2 \leq j \leq m-1$, and $j=m$ in (34) are discussed separately.

Upper Bound of $\lambda_{1}(Q)$. From (19), we have

$$
\lambda_{1}(S) \leq \min \left\{\widetilde{d}_{1}+t, \widetilde{d}_{2}, \widetilde{d}_{m}+s\right\},
$$

where $\tilde{d}_{k}, s$, and $t$ are functions of $\rho$ on the interval $[0,1]$. In this case, we consider $\rho$ in the following four subintervals: $\left[0, d_{1} / d_{m}\right],\left[d_{1} / d_{m}, d_{2} / d_{m}\right],\left[d_{2} / d_{m}, d_{m-1} / d_{m}\right]$, and $\left[d_{m-1} / d_{m}, 1\right]$, respectively. For $\rho \in\left[0, d_{1} / d_{m}\right]$, we have $\tilde{d}_{1}+t=f_{4}(\rho), \tilde{d}_{2}=d_{1}$, and $\tilde{d}_{m}+s=f_{1}(\rho)$. For $\rho \in$ $\left[d_{1} / d_{m}, d_{2} / d_{m}\right]$, we have $\tilde{d}_{1}+t=d_{1}+f_{2}(\rho), \tilde{d}_{2}=\rho d_{m}$, and $\widetilde{d}_{m}+s=d_{m-1}+f_{1}(\rho)$. For $\rho \in\left[d_{2} / d_{m}, d_{m-1} / d_{m}\right]$, we have $\widetilde{d}_{1}+t=d_{1}+f_{2}(\rho), \widetilde{d}_{2}=d_{2}$, and $\widetilde{d}_{m}+s=d_{m-1}+f_{1}(\rho)$. For $\rho \in\left[d_{m-1} / d_{m}, 1\right]$, we have $\tilde{d}_{1}+t=d_{1}+f_{2}(\rho), \tilde{d}_{2}=d_{2}$, and $\widetilde{d}_{m}+s=f_{3}(\rho)$. Hence

$$
\begin{aligned}
& \min _{\rho \in[0,1]} \tilde{d}_{2}=d_{1}, \\
& \min _{\rho \in V}\left(\tilde{d}_{m}+s\right)= \begin{cases}d_{m-1}+f_{1}\left(\frac{d_{1}}{d_{m}}\right), & \text { if } V=\left[0, \frac{d_{1}}{d_{m}}\right], \\
d_{m-1}+f_{1}\left(\frac{d_{2}}{d_{m}}\right), & \text { if } V=\left[\frac{d_{1}}{d_{m}}, \frac{d_{2}}{d_{m}}\right], \\
d_{m-1}+f_{1}\left(\frac{d_{m-1}}{d_{m}}\right), & \text { if } V=\left[\frac{d_{2}}{d_{m}}, \frac{d_{m-1}}{d_{m}}\right], \\
d_{m-1}+f_{1}\left(\frac{d_{m-1}}{d_{m}}\right), & \text { if } V=\left[\frac{d_{m-1}}{d_{m}}, 1\right],\end{cases} \\
& \min _{\rho \in V}\left(\tilde{d}_{1}+t\right)= \begin{cases}f_{2}(0), & \text { if } V=\left[0, \frac{d_{1}}{d_{m}}\right], \\
d_{1}+f_{2}\left(\frac{d_{2}}{d_{m}}\right), & \text { if } V=\left[\frac{d_{1}}{d_{m}}, \frac{d_{2}}{d_{m}}\right], \\
d_{1}+f_{2}\left(\frac{d_{m-1}}{d_{m}}\right), & \text { if } V=\left[\frac{d_{2}}{d_{m}}, \frac{d_{m-1}}{d_{m}}\right], \\
d_{1}+f_{2}(1), & \text { if } V=\left[\frac{d_{m-1}}{d_{m}}, 1\right] .\end{cases}
\end{aligned}
$$

Since $0>f_{1}\left(d_{1} / d_{m}\right)>f_{1}\left(d_{2} / d_{m}\right)>f_{1}\left(d_{m-1} / d_{m}\right), f_{2}(0) \geq$ $d_{m}>d_{1}$, and $f_{2}\left(d_{2} / d_{m}\right)>f_{2}\left(d_{m-1} / d_{m}\right)>f_{2}(1)>0$, we have

$$
\lambda_{1}(Q) \leq \min \left\{d_{1}, d_{m-1}+f_{1}\left(\frac{d_{m-1}}{d_{m}}\right)\right\} .
$$

Upper Bound of $\lambda_{j}(Q)$, for $2 \leq j \leq m-1$. From (19), we have

$$
\lambda_{j}(Q) \leq \min \left\{\tilde{d}_{j}+t, \tilde{d}_{j+1}\right\} .
$$


In this case, we consider $\rho$ lying in the following four subintervals: $\left[0, d_{j-1} / d_{m}\right],\left[d_{j-1} / d_{m}, d_{j} / d_{m}\right],\left[d_{j} / d_{m}, d_{j+1} / d_{m}\right]$, and $\left[d_{j+1} / d_{m}, 1\right]$, respectively. For $\rho \in\left[0, d_{j-1} / d_{m}\right]$, we have $\tilde{d}_{j}+$ $t=d_{j-1}+f_{2}(\rho)$ and $\tilde{d}_{j+1}=d_{j}$. For $\rho \in\left[d_{j-1} / d_{m}, d_{j} / d_{m}\right]$, we have $\tilde{d}_{j}+t=f_{4}(\rho)$ and $\tilde{d}_{j+1}=d_{j}$. For $\rho \in\left[d_{j} / d_{m}, d_{j+1} / d_{m}\right]$, we have $\tilde{d}_{j}+t=d_{j}+f_{2}(\rho)$ and $\tilde{d}_{j+1}=\rho d_{m}$. For $\rho \in$ $\left[d_{j+1} / d_{m}, 1\right]$, we have $\tilde{d}_{j}+t=d_{j}+f_{2}(\rho)$ and $\tilde{d}_{j+1}=d_{j+1}$. Hence

$$
\min _{\rho \in V}\left(\tilde{d}_{j}+t\right)= \begin{cases}d_{j-1}+f_{2}\left(\frac{d_{j-1}}{d_{m}}\right), & \text { if } V=\left[0, \frac{d_{j-1}}{d_{m}}\right], \\ d_{j-1}+f_{2}\left(\frac{d_{j-1}}{d_{m}}\right), & \text { if } V=\left[\frac{d_{j-1}}{d_{m}}, \frac{d_{j}}{d_{m}}\right], \\ d_{j}+f_{2}\left(\frac{d_{j+1}}{d_{m}}\right), & \text { if } V=\left[\frac{d_{j}}{d_{m}}, \frac{d_{j+1}}{d_{m}}\right], \\ d_{j}+f_{2}(1), & \text { if } V=\left[\frac{d_{j-1}}{d_{m}}, 1\right] .\end{cases}
$$

Therefore,

$$
\lambda_{j}(Q) \leq \min \left\{d_{j-1}+f_{2}\left(\frac{d_{j-1}}{d_{m}}\right), d_{j}\right\} .
$$

Since $d_{j-1}+f_{2}\left(d_{j-1} / d_{m}\right)=f_{4}\left(d_{j-1} / d_{m}\right)>f_{4}(0) \geq d_{m} \geq d_{j}$, we get

$$
\lambda_{j}(Q) \leq d_{j}
$$

Upper Bound of $\lambda_{m}(Q)$. From (19) we have

$$
\lambda_{m}(Q) \leq \tilde{d}_{m}+t
$$

For $\rho \in\left[0, d_{m-1} / d_{m}\right]$, we have $\tilde{d}_{m}+t=d_{m-1}+f_{2}(\rho)$ while for $\rho \in\left[d_{m-1} / d_{m}, 1\right]$, we have $\tilde{d}_{m}+t=f_{4}(\rho)$ :

$$
\min _{\rho \in V}\left(\tilde{d}_{m}+t\right)= \begin{cases}d_{m-1}+f_{2}\left(\frac{d_{m-1}}{d_{m}}\right), & \text { if } V=\left[0, \frac{d_{m-1}}{d_{m}}\right], \\ d_{m-1}+f_{2}\left(\frac{d_{m-1}}{d_{m}}\right), & \text { if } V=\left[\frac{d_{m-1}}{d_{m}}, 1\right] .\end{cases}
$$

Hence,

$$
\lambda_{m}(Q) \leq d_{m-1}+f_{2}\left(\frac{d_{m-1}}{d_{m}}\right)
$$

This completes the proof.
Theorem 12. Let $Q$ be an irreducible arrowhead matrix defined by (16) and satisfying all assumptions in Theorem 8. Then the eigenvalues of $Q$ are bounded below by

$$
\lambda_{j}(Q) \geq \begin{cases}d_{1}+f_{1}\left(\frac{d_{1}}{d_{m}}\right), & \text { if } j=1, \\ \max \left\{d_{j-1}, d_{j}+f_{1}\left(\frac{d_{j}}{d_{m}}\right)\right\}, & \text { if } 2 \leq j \leq m-1, \\ d_{1}+f_{2}\left(\frac{d_{1}}{d_{m}}\right), & \text { if } j=m .\end{cases}
$$

Proof. In Theorem 8, the lower bounds of the eigenvalues of $Q$ in (20) are determined by $\tilde{d}_{j}, j=1,2, \ldots, m$, and $s$ and $t$ in (21). As we did in Theorem 12, the lower bounds of the eigenvalues of $Q$ are functions of $\rho$ in the interval $[0,1]$. Therefore, we are able to find optimal bounds of the eigenvalues of $Q$ by choosing proper $\rho$. The discussion is given for $j=1,2 \leq j \leq m-1$, and $j=m$ in (45), separately.

Lower Bound of $\lambda_{1}(Q)$. From (20), we have

$$
\lambda_{1}(Q) \geq \tilde{d}_{1}+s .
$$

In this case, we consider $\rho$ lying in the following two subintervals: $\left[0, d_{1} / d_{m}\right]$ and $\left[d_{1} / d_{m}, 1\right]$. For $\rho \in\left[0, d_{1} / d_{m}\right]$, $\tilde{d}_{1}+s=f_{3}(\rho)$. For $\rho \in\left[d_{1} / d_{m}, 1\right]$, we have $\tilde{d}_{1}+s=d_{1}+f_{1}(\rho)$. Hence

$$
\max _{\rho \in V}\left(\tilde{d}_{1}+s\right)= \begin{cases}d_{1}+f_{1}\left(\frac{d_{1}}{d_{m}}\right), & \text { if } V=\left[0, \frac{d_{1}}{d_{m}}\right], \\ d_{1}+f_{1}\left(\frac{d_{1}}{d_{m}}\right), & \text { if } V=\left[\frac{d_{1}}{d_{m}}, 1\right] .\end{cases}
$$

It leads to

$$
\lambda_{1}(Q) \geq d_{1}+f_{1}\left(\frac{d_{1}}{d_{m}}\right)
$$

Lower Bound of $\lambda_{2}(Q)$. From (20), we have

$$
\lambda_{2}(Q) \geq \max \left\{\tilde{d}_{1}, \tilde{d}_{2}+s\right\} .
$$

In this case, we consider $\rho$ lying in the following three subintervals: $\left[0, d_{1} / d_{m}\right],\left[d_{1} / d_{m}, d_{2} / d_{m}\right]$, and $\left[d_{2} / d_{m}, 1\right]$. For $\rho \in\left[0, d_{1} / d_{m}\right]$, we have $\tilde{d}_{1}=\rho d_{m}, \tilde{d}_{2}+s=d_{1}+f_{1}(\rho)$. For $\rho \in\left[d_{1} / d_{m}, d_{2} / d_{m}\right]$, we have $\tilde{d}_{1}=d_{1}, \tilde{d}_{2}+s=f_{3}(\rho)$. For $\rho \in\left[d_{2} / d_{m}, 1\right]$, we have $\tilde{d}_{1}=d_{1}$ and $\tilde{d}_{2}+s=d_{2}+f_{1}(\rho)$. Hence,

$$
\max _{\rho \in V}\left(\tilde{d}_{2}+s\right)= \begin{cases}d_{1}+f_{1}(0), & \text { if } V=\left[0, \frac{d_{1}}{d_{m}}\right], \\ d_{2}+f_{1}\left(\frac{d_{2}}{d_{m}}\right), & \text { if } V=\left[\frac{d_{1}}{d_{m}}, \frac{d_{2}}{d_{m}}\right], \\ d_{2}+f_{1}\left(\frac{d_{2}}{d_{m}}\right), & \text { if } V=\left[\frac{d_{2}}{d_{m}}, 1\right] .\end{cases}
$$


These lead to

$$
\lambda_{2}(Q) \geq \max \left\{d_{1}, d_{2}+f_{1}\left(\frac{d_{2}}{d_{m}}\right)\right\} .
$$

Lower Bound of $\lambda_{j}(Q), 3 \leq j \leq m-1$. From (20), we have

$$
\lambda_{j}(Q) \geq \max \left\{\tilde{d}_{j-1}, \tilde{d}_{j}+s\right\} .
$$

In this case, we consider $\rho$ lying in the following three subintervals: $\left[0, d_{j-2} / d_{m}\right],\left[d_{j-2} / d_{m}, d_{j-1} / d_{m}\right],\left[d_{j-1} / d_{m}, d_{j} / d_{m}\right]$, and $\left[d_{j} / d_{m}, 1\right]$. For $\rho \in\left[0, d_{j-2} / d_{m}\right]$, we have $\tilde{d}_{j-1}=d_{j-2}$ and $\tilde{d}_{j}+s=d_{j-1}+f_{1}(\rho)$. For $\rho \in\left[d_{j-2} / d_{m}, d_{j-1} / d_{m}\right]$, we have $\tilde{d}_{j-1}=\rho d_{m}$ and $\tilde{d}_{j}+s=d_{j-1}+f_{1}(\rho)$. For $\rho \in$ $\left[d_{j-1} / d_{m}, d_{j} / d_{m}\right]$, we have $\tilde{d}_{j-1}=d_{j-1}$ and $\tilde{d}_{j}+s=f_{3}(\rho)$. For $\rho \in\left[d_{j} / d_{m}, 1\right]$, we have $\tilde{d}_{j-1}=d_{j-1}$ and $\tilde{d}_{j}+s=d_{j}+f_{1}(\rho)$. Hence

$$
\max _{\rho \in V}\left(\tilde{d}_{j \in V}+s\right)= \begin{cases}\max _{j-1}+f_{1}(0), & \text { if } V=\left[0, \frac{d_{j-2}}{d_{m}}\right], \\ d_{j-1}+f_{1}\left(\frac{d_{j-2}}{d_{m}}\right), & \text { if } V=\left[\frac{d_{j-2}}{d_{m}}, \frac{d_{j-1}}{d_{m}}\right], \\ d_{j}+f_{1}\left(\frac{d_{j}}{d_{m}}\right), & \text { if } V=\left[\frac{d_{j-1}}{d_{m}}, \frac{d_{j}}{d_{m}}\right], \\ d_{j}+f_{1}\left(\frac{d_{j}}{d_{m}}\right), & \text { if } V=\left[\frac{d_{j}}{d_{m}}, 1\right] .\end{cases}
$$

Since $d_{j-1}>d_{j-1}+f_{1}(0)>d_{j-1}+f_{1}\left(d_{j-2} / d_{m}\right)$, we have

$$
\lambda_{j}(Q) \geq \max \left\{d_{j-1}, d_{j}+f_{1}\left(\frac{d_{j}}{d_{m}}\right)\right\} .
$$

Lower Bound of $\lambda_{m}(Q)$. From (20), we have

$$
\lambda_{m}(Q) \geq \max \left\{\tilde{d}_{1}+t, \tilde{d}_{m-1}, \tilde{d}_{m}+s\right\} .
$$

In this case, we consider $\rho$ lying in the following three subintervals: $\left[0, d_{1} / d_{m}\right],\left[d_{1} / d_{m}, d_{m-2} / d_{m}\right],\left[d_{m-2} / d_{m}, d_{m-1} / d_{m}\right]$, and $\left[d_{m-1} / d_{m}, 1\right]$. For $\rho \in\left[0, d_{1} / d_{m}\right]$, we have $\tilde{d}_{1}+t=$ $f_{4}(\rho), \tilde{d}_{m-1}=d_{m-2}, \tilde{d}_{m}+s=d_{m-1}+f_{1}(\rho)$. For $\rho \in$ $\left[d_{1} / d_{m}, d_{m-2} / d_{m}\right]$, we have $\tilde{d}_{1}+t=d_{1}+f_{2}(\rho), \widetilde{d}_{m-1}=d_{m-2}$, $\tilde{d}_{m}+s=d_{m-1}+f_{1}(\rho)$. For $\rho \in\left[d_{m-2} / d_{m}, d_{m-1} / d_{m}\right]$, we have $\tilde{d}_{1}+t=d_{1}+f_{2}(\rho), \tilde{d}_{m-1}=\rho d_{m}, \tilde{d}_{m}+s=d_{m-1}+f_{1}(\rho)$. For $\rho \in\left[d_{m-1} / d_{m}, 1\right]$, we have $\tilde{d}_{1}+t=d_{1}+f_{2}(\rho), \tilde{d}_{m-1}=d_{m-1}$, $\tilde{d}_{m}+s=f_{3}(\rho)$. Hence

$$
\begin{aligned}
& \max _{\rho \in[0,1]} \tilde{d}_{m-1}=d_{m-1}, \\
& \max _{\rho \in V}\left(\tilde{d}_{m}+s\right)= \begin{cases}d_{m-1}+f_{1}(0), & \text { if } V=\left[0, \frac{d_{1}}{d_{m}}\right], \\
d_{m-1}+f_{1}\left(\frac{d_{1}}{d_{m}}\right), & \text { if } V=\left[\frac{d_{1}}{d_{m}}, \frac{d_{m-2}}{d_{m}}\right], \\
d_{m-1}+f_{1}\left(\frac{d_{m-2}}{d_{m}}\right), & \text { if } V=\left[\frac{d_{m-2}}{d_{m}}, \frac{d_{m-1}}{d_{m}}\right], \\
d_{m}+f_{1}(1), & \text { if } V=\left[\frac{d_{m-1}}{d_{m}}, 1\right],\end{cases} \\
& \max _{\rho \in V}\left(\tilde{d}_{1}+t\right)= \begin{cases}d_{1}+f_{2}\left(\frac{d_{1}}{d_{m}}\right), & \text { if } V=\left[0, \frac{d_{1}}{d_{m}}\right], \\
d_{1}+f_{2}\left(\frac{d_{1}}{d_{m}}\right), & \text { if } V=\left[\frac{d_{1}}{d_{m}}, \frac{d_{m-2}}{d_{m}}\right], \\
d_{1}+f_{2}\left(\frac{d_{m-2}}{d_{m}}\right), & \text { if } V=\left[\frac{d_{m-2}}{d_{m}}, \frac{d_{m-1}}{d_{m}}\right], \\
d_{1}+f_{2}\left(\frac{d_{m-1}}{d_{m}}\right), & \text { if } V=\left[\frac{d_{m-1}}{d_{m}}, 1\right] .\end{cases}
\end{aligned}
$$

Since $0>f_{1}(0)>f_{1}\left(d_{1} / d_{m}\right)>f_{1}\left(d_{m-2} / d_{m}\right)$ and $f_{2}\left(d_{1} / d_{m}\right)>$ $f_{2}\left(d_{m-2} / d_{m}\right)>f_{2}\left(d_{m-1} / d_{m}\right)$, we have

$$
\lambda_{m}(Q) \geq \max \left\{d_{m-1}, d_{m}+f_{1}(1), d_{1}+f_{2}\left(\frac{d_{1}}{d_{m}}\right)\right\} .
$$

Since $d_{1}+f_{2}\left(d_{1} / d_{m}\right)=f_{4}\left(d_{1} / d_{m}\right)>f_{4}(0) \geq d_{m}$, we get

$$
\lambda_{m}(Q) \geq d_{1}+f_{2}\left(\frac{d_{1}}{d_{m}}\right)
$$

This completes the proof.

Corollary 13. Let $Q$ be an irreducible arrowhead matrix defined by (16) and satisfying all assumption in Theorem 8. Then upper and lower bounds of the eigenvalues of $Q$ obtained by Theorems 11 and 12 are tighter than those given by (2), (3), and (4).

Proof. Since

$$
\min \left\{d_{1}, d_{m-1}+f_{1}\left(\frac{d_{m-1}}{d_{m}}\right)\right\} \leq d_{1},
$$

then the upper bound for the eigenvalue $\lambda_{1}(Q)$ given by (34) in Theorem 11 is tighter than that by (2). The upper bounds for the eigenvalues $\lambda_{j}(Q), j=2, \ldots, m-1$, provided by (34) in Theorem 11 are the same as those by (2). 
Note that $0 \leq d_{1}<\cdots<d_{m-1} \leq d_{m}$; the right-hand side of (3) with $b=d_{m}$ is

$$
\begin{aligned}
\max & \left\{d_{1}+\left|c_{1}\right|, \ldots, d_{m-1}+\left|c_{m-1}\right|, b+\sum_{i=1}^{m-1}\left|c_{i}\right|\right\} \\
& =d_{m}+\sum_{i=1}^{m-1}\left|c_{i}\right| .
\end{aligned}
$$

Since $\|c\| \leq \sum_{i=1}^{m-1}\left|c_{i}\right|, d_{m}+\|c\|=f_{4}(1)$, and $d_{m-1}+$ $f_{2}\left(d_{m-1} / d_{m}\right)=f_{4}\left(d_{m-1} / d_{m}\right)$, we have

$d_{m}+\sum_{i=1}^{m-1}\left|c_{i}\right|-\left[d_{m-1}+f_{2}\left(\frac{d_{m-1}}{d_{m}}\right)\right] \geq f_{4}(1)-f_{4}\left(\frac{d_{m-1}}{d_{m}}\right)>0$,

and then the upper bound of $\lambda_{m}(Q)$ from (34) in Theorem 11 is tighter than that from (3).

Now we turn to the lower bounds of $\lambda_{j}(Q)$. Since

$$
\max \left\{d_{j-1}, d_{j}+f_{1}\left(\frac{d_{j}}{d_{m}}\right)\right\} \geq d_{j-1}
$$

for $j=2, \ldots, m-1$ and

$$
d_{1}+f_{2}\left(\frac{d_{1}}{d_{m}}\right) \geq d_{m}>d_{m-1}
$$

we know that the lower bounds for the eigenvalues $\lambda_{j}(Q)$, $j=2, \ldots, m$, provided by (45) in Theorem 12 are tighter than those by (2).

Remark 14. When $c$ in (16) is a zero vector, by using Theorems 11 and 12, we have $d_{j} \leq \lambda(Q) \leq d_{j}$, that is, $\lambda(Q)=d_{j}$. In this sense, the lower and upper bounds given in Theorems 11 and 12 are sharp.

Remark 15. When $Q$ in Theorems 11 and 12 has size of $2 \times 2$, the upper and lower bounds of its each eigenvalue are identical. Actually, from Theorems 11 and 12 we have

$$
\begin{gathered}
d_{1}+f_{1}\left(\frac{d_{1}}{d_{2}}\right) \leq \lambda_{1}(Q) \leq \min \left\{d_{1}, d_{1}+f_{1}\left(\frac{d_{1}}{d_{2}}\right)\right\}, \\
d_{1}+f_{2}\left(\frac{d_{1}}{d_{2}}\right) \leq \lambda_{2}(Q) \leq d_{1}+f_{2}\left(\frac{d_{1}}{d_{2}}\right) .
\end{gathered}
$$

Clearly, we have

$$
\lambda_{1}(Q)=d_{1}+f_{1}\left(\frac{d_{1}}{d_{2}}\right), \quad \lambda_{2}(Q)=d_{1}+f_{2}\left(\frac{d_{1}}{d_{2}}\right) .
$$

This can be verified by calculating the eigenvalues $Q$ directly.

Remark 16. For the lower bound of the smallest eigenvalue of an arrowhead matrix, no conclusion can be made for the tightness of the bounds by using (4) and (45) in Theorem 12. An example will be given later (see Example 22 in Section 5).

\section{Hub Matrices}

Using the improved upper and lower bounds for the arrowhead matrix, we will now examine their applications to hub matrices and MIMO wireless communication systems. The concept of the hub matrix was introduced in the context of wireless communications by Kung and Suter in [9] and it is reexamined here.

Definition 17. A matrix $A \in \mathbb{R}^{n \times m}$ is called a hub matrix, if its first $m-1$ columns (called nonhub columns) are orthogonal to each other with respect to the Euclidean inner product and its last column (called hub column) has its Euclidean norm greater than or equal to that of any other columns. We assume that all columns of $A$ are nonzeros vectors.

We denote the columns of a hub matrix $A$ by $a_{1}, a_{2}, \ldots, a_{m}$. Vectors $a_{1}, a_{2}, \ldots, a_{m-1}$ are orthogonal to each other. We further assume that $0<\left\|a_{1}\right\| \leq\left\|a_{2}\right\| \leq \cdots \leq$ $\left\|a_{m}\right\|$. In such case, we call $A$ an ordered hub matrix. Our interest is to study the eigenvalues of $Q=A^{t} A$, the Gram matrix $A$. In the context of wireless communication systems, $Q$ is also called the system matrix. The matrix $Q$ has a form as follows:

$$
Q=\left[\begin{array}{ccccc}
\left\|a_{1}\right\|^{2} & & & & \left\langle a_{1}, a_{m}\right\rangle \\
& \left\|a_{2}\right\|^{2} & & & \left\langle a_{2}, a_{m}\right\rangle \\
& & \ddots & & \vdots \\
& & & \left\|a_{m-1}\right\|^{2} & \left\langle a_{m-1}, a_{m}\right\rangle \\
& & & & \\
\left\langle a_{m}, a_{1}\right\rangle & \left\langle a_{m}, a_{2}\right\rangle & \cdots & \left\langle a_{m}, a_{m-1}\right\rangle & \left\|a_{m}\right\|^{2}
\end{array}\right] .
$$

Clearly, $Q$ is an arrowhead matrix associated with $A$.

An important way to characterize properties of $Q$ is in terms of ratios of its successive eigenvalues. To this end, the ratios are called eigengap of $Q$ which are defined [9] to be

$$
\mathrm{EG}_{i}(Q)=\frac{\lambda_{m-(i-1)}(Q)}{\lambda_{m-i}(Q)}
$$

for $i=1,2, \ldots, m-1$. Following the definition in [9], we define the $i$ th hub-gap of $A$ as follows:

$$
\mathrm{HG}_{i}(A)=\frac{\left\|a_{m-(i-1)}\right\|^{2}}{\left\|a_{m-i}\right\|^{2}}
$$

for $i=1,2, \ldots, m-1$.

The hub-gaps of $A$ will allow us to predict the eigenstructure of $Q$. It was shown in [9] that the lower and upper bounds of $\mathrm{EG}_{1}(Q)$ [9] are given by the following:

$$
\mathrm{HG}_{1}(A) \leq \mathrm{EG}_{1}(Q) \leq\left(\mathrm{HG}_{1}(A)+1\right) \mathrm{HG}_{2}(A)
$$

These bounds only involve nonhub columns having the two largest Euclidean norms and the hub column of $A$. Using 
the results in Theorems 11 and 12, we obtain the following bounds:

$$
\begin{aligned}
& \frac{f_{4}\left(\left\|a_{1}\right\|^{2} /\left\|a_{m}\right\|^{2}\right)}{\left\|a_{m-1}\right\|^{2}} \\
& \quad \leq \mathrm{EG}_{1}(Q) \leq \frac{f_{4}\left(\left\|a_{m-1}\right\|^{2} /\left\|a_{m}\right\|^{2}\right)}{\max \left\{\left\|a_{m-2}\right\|^{2}, f_{3}\left(\left\|a_{m-1}\right\|^{2} /\left\|a_{m}\right\|^{2}\right)\right\}} .
\end{aligned}
$$

Obviously, these bounds are not only related to two nonhub columns with the largest Euclidean norms and the hub column of $A$ but also related to the nonhub column having the smallest Euclidean norm and interrelationship between all nonhub columns and the hub column of $A$. As we expected, the lower and upper bounds of $\mathrm{EG}_{1}(Q)$ in (70) should be tighter than those in (69). To prove this statement, we give the following lemma first.

Lemma 18. Let $a_{1}, a_{2}, \ldots, a_{m}$ be the columns of a hub matrix A with $0<\left\|a_{1}\right\| \leq\left\|a_{2}\right\| \leq \cdots \leq\left\|a_{m-1}\right\| \leq\left\|a_{m}\right\|$. Then

$$
\begin{gathered}
f_{4}(\rho)>\left\|a_{m}\right\|^{2} \quad \text { for } \rho \in(0,1], \\
f_{4}\left(\frac{\left\|a_{m-1}\right\|^{2}}{\left\|a_{m}\right\|^{2}}\right)<\left\|a_{m}\right\|^{2}+\left\|a_{m-1}\right\|^{2} .
\end{gathered}
$$

Proof. From Lemma 10, we know, for $\rho \in(0,1]$,

$$
f_{4}(\rho)>f_{4}(0)=\frac{\left\|a_{m}\right\|^{2}+\sqrt{\left\|a_{m}\right\|^{4}+4\|c\|^{2}}}{2}>\left\|a_{m}\right\|^{2}
$$

where $\|c\|^{2}=\sum_{i=1}^{m-1}\left|\left\langle a_{i}, a_{m}\right\rangle\right|^{2}$. The inequality (71) holds. By the definition of $f_{4}$, showing the inequality (72) is equivalent to proving

$$
\|c\|^{2} \leq\left\|a_{m}\right\|^{2}\left\|a_{m-1}\right\|^{2}
$$

This is true because

$$
\begin{aligned}
\left\|a_{m}\right\|^{2} & \geq \sum_{j=1}^{m-1} \frac{1}{\left\|a_{j}\right\|^{2}}\left|\left\langle a_{j}, a_{m}\right\rangle\right|^{2} \\
& \geq \sum_{j=1}^{m-1} \frac{1}{\left\|a_{m-1}\right\|^{2}}\left|\left\langle a_{j}, a_{m}\right\rangle\right|^{2}=\frac{\|c\|^{2}}{\left\|a_{m-1}\right\|^{2}} .
\end{aligned}
$$

The first inequality of above is from the orthogonality of $a_{j}$, $j=1, \ldots, m-1$ while the second inequality is from $\left\|a_{1}\right\| \leq$ $\left\|a_{2}\right\| \leq \cdots \leq\left\|a_{m-1}\right\|$. This completes the proof.

The following result holds.

Proposition 19. Let $Q$ in (66) be the arrowhead matrix associated with a hub matrix A. Assume that $0<\left\|a_{1}\right\|<$ $\left\|a_{2}\right\|<\cdots<\left\|a_{m-1}\right\| \leq\left\|a_{m}\right\|$, where $a_{j}, j=1, \ldots, m$ are columns of $A$. Then the bounds of the $E G_{1}(Q)$ in (70) are tighter than those in (69).
Proof. We first need to show

$$
\frac{\left\|a_{m}\right\|^{2}}{\left\|a_{m-1}\right\|^{2}}<\frac{f_{4}\left(\left\|a_{1}\right\|^{2} /\left\|a_{m}\right\|^{2}\right)}{\left\|a_{m-1}\right\|^{2}} .
$$

Clearly, this is true because of (71). Next we need to show

$$
\begin{gathered}
\frac{f_{4}\left(\left\|a_{m-1}\right\|^{2} /\left\|a_{m}\right\|^{2}\right)}{\max \left\{\left\|a_{m-2}\right\|^{2}, f_{3}\left(\left\|a_{m-1}\right\|^{2} /\left\|a_{m}\right\|^{2}\right)\right\}} \\
<\left(\frac{\left\|a_{m}\right\|^{2}}{\left\|a_{m-1}\right\|^{2}}+1\right) \frac{\left\|a_{m-1}\right\|^{2}}{\left\|a_{m-2}\right\|^{2}} .
\end{gathered}
$$

To this end, it is suffice to prove

$$
f_{4}\left(\frac{\left\|a_{m-1}\right\|^{2}}{\left\|a_{m}\right\|^{2}}\right)<\left\|a_{m}\right\|^{2}+\left\|a_{m-1}\right\|^{2} .
$$

This is exactly (72). The proof is complete.

The lower bound in (70) can be rewritten in terms of the hubgap of $A$ as follows:

$$
\frac{f_{4}\left(\left\|a_{1}\right\|^{2} /\left\|a_{m}\right\|^{2}\right)}{\left\|a_{m-1}\right\|^{2}}=\frac{1}{2} \mathrm{HG}_{1}(Q)\left[1+\sqrt{1+\frac{4\|c\|^{2}}{\left\|a_{m}\right\|^{4}}}\right]
$$

The upper bound in (70) can be rewritten in terms of the hubgap of $A$ as follows:

$$
\begin{aligned}
& \frac{f_{4}\left(\left\|a_{m-1}\right\|^{2} /\left\|a_{m}\right\|^{2}\right)}{\max \left\{\left\|a_{m-2}\right\|^{2}, f_{3}\left(\left\|a_{m-1}\right\|^{2} /\left\|a_{m}\right\|^{2}\right)\right\}} \\
& \leq \frac{f_{4}\left(\left\|a_{m-1}\right\|^{2} /\left\|a_{m}\right\|^{2}\right)}{\left\|a_{m-2}\right\|^{2}} \\
& =\frac{1}{2}\left(\mathrm{HG}_{1}(A)+1\right) \mathrm{HG}_{2}(A) \\
& +\frac{1}{2}\left(\mathrm{HG}_{1}(A)-1\right) \mathrm{HG}_{2}(A) \sqrt{1+\frac{4\|c\|^{2}}{\left(\left\|a_{m}\right\|^{2}-\left\|a_{m-1}\right\|^{2}\right)^{2}}} .
\end{aligned}
$$

To compare these bounds to Kung and Suter [9], set $\|c\|^{2}=$ 0 , and the bounds for $\operatorname{EigGap}_{1}(Q)$ in (70) become

$$
\mathrm{HG}_{1}(A) \leq \mathrm{EG}_{1}(Q) \leq \mathrm{HG}_{1}(A) \mathrm{HG}_{2}(A) \text {. }
$$

Under these conditions, the lower bound agrees with Kung and Suter while the upper bound is tighter.

Let $A \in \mathbb{R}^{n \times m}$ be an ordered hub matrix. Let $\tilde{A} \in$ $\mathbb{R}^{n \times k}$ be a hub matrix obtained by removing the first $n-k$ nonhub columns of $A$ with the smallest Euclidean norms. This corresponds to the MIMO beamforming problem by comparing $k$ of $m$ transmitting antennas with the largest signal-to-noise ratio (see [9]). The ratio $\lambda_{k}(\widetilde{Q}) / \lambda_{m}(Q)$ with 
$\widetilde{Q}=\tilde{A}^{t} \tilde{A}$ describes the relative performance of the resulting systems. It was shown in [9] that for $k \geq 2$

$$
\frac{\left\|a_{m}\right\|^{2}}{\left\|a_{m}\right\|^{2}+\left\|a_{m-1}\right\|^{2}} \leq \frac{\lambda_{k}(\tilde{Q})}{\lambda_{m}(Q)} \leq \frac{\left\|a_{m}\right\|^{2}+\left\|a_{m-1}\right\|^{2}}{\left\|a_{m}\right\|^{2}} .
$$

By Theorems 11 and 12, we have

$$
\frac{f_{4}\left(\left\|a_{m-k+1}\right\|^{2} /\left\|a_{m}\right\|^{2}\right)}{f_{4}\left(\left\|a_{m-1}\right\|^{2} /\left\|a_{m}\right\|^{2}\right)} \leq \frac{\lambda_{k}(\tilde{Q})}{\lambda_{m}(Q)} \leq \frac{f_{4}\left(\left\|a_{m-1}\right\|^{2} /\left\|a_{m}\right\|^{2}\right)}{f_{4}\left(\left\|a_{1}\right\|^{2} /\left\|a_{m}\right\|^{2}\right)} \text {. }
$$

By Lemma 18, the lower and upper bounds for the ratio $\lambda_{k}(\widetilde{Q}) / \lambda_{m}(Q)$ in $(83)$ are better than those in (82). In particular, when $k=1$, the matrix $\widetilde{Q}$ corresponds to the hub, as such, it reduces to $\widetilde{Q}=\left[\left\|a_{m}\right\|^{2}\right]$; hence, $\lambda_{1}(\widetilde{Q})=$ $\left\|a_{m}\right\|^{2}$. Therefore, an estimate of the quantity $\left\|a_{m}\right\|^{2} / \lambda_{m}(Q)$ was given in [9] as follows:

$$
\frac{\left\|a_{m}\right\|^{2}}{\left\|a_{m}\right\|^{2}+\left\|a_{m-1}\right\|^{2}} \leq \frac{\left\|a_{m}\right\|^{2}}{\lambda_{m}(Q)} \leq 1 .
$$

By Theorems 11 and 12, we have

$$
\frac{\left\|a_{m}\right\|^{2}}{f_{4}\left(\left\|a_{m-1}\right\|^{2} /\left\|a_{m}\right\|^{2}\right)} \leq \frac{\left\|a_{m}\right\|^{2}}{\lambda_{m}(Q)} \leq \frac{\left\|a_{m}\right\|^{2}}{f_{4}\left(\left\|a_{1}\right\|^{2} /\left\|a_{m}\right\|^{2}\right)} .
$$

Again, by Lemma 18, the lower and upper bounds for the ratio $\left\|a_{m}\right\|^{2} / \lambda_{m}(Q)$ in (85) are better than those in (84). We can simply view (84) and (85) as degenerate forms of (82) and (83), respectively.

\section{Numerical Examples}

In this section, we will numerically compare the lower and upper bounds of eigenvalues for arrowhead matrices estimated by three approaches. The first approach is due to Cauchy, denoted by $C$ and based on (2)-(4). The second approach, denoted by SS, is based on eigenvalue bounds provided by Theorems 11 and 12. The third approach, denoted by WS, is based on Wolkowicz-Styan's lower and upper bounds for the largest and smallest eigenvalues of a symmetric matrix [15]. These WS bounds are given by

$$
\begin{aligned}
& a-s p \leq \lambda_{1}(Q) \leq a-\frac{s}{p}, \\
& a+\frac{s}{p} \leq \lambda_{m}(Q) \leq a+s p,
\end{aligned}
$$

where $Q \in \mathbb{R}^{m \times m}$ is symmetric, $p=\sqrt{m-1}, a=$ $\operatorname{trace}(Q) / m$, and $s^{2}=\operatorname{trace}\left(Q^{t} Q\right) / m-a^{2}$.

Example 20. Consider the directed graph in Figure 1, which might be used to represent a MIMO communication scheme.

The adjacency matrix for the directed graph is

$$
A=\left[\begin{array}{llll}
0 & 1 & 0 & 1 \\
0 & 0 & 1 & 1 \\
0 & 1 & 0 & 1 \\
1 & 0 & 0 & 1
\end{array}\right],
$$

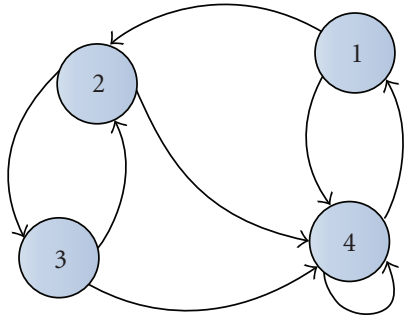

Figure 1: A directed graph.

which is a hub matrix with the right-most column corresponding to node 4 as the hub column. The associated arrowhead matrix $Q=A^{T} A$ is

$$
Q=\left[\begin{array}{llll}
1 & 0 & 0 & 1 \\
0 & 2 & 0 & 2 \\
0 & 0 & 1 & 1 \\
1 & 2 & 1 & 4
\end{array}\right] .
$$

The eigenvalues of $Q$ are $0,1,1.4384,5.5616$. Corollary 3 implies 1 being the eigenvalue of $Q$. By Corollary 4, the following matrix

$$
\widetilde{Q}=\left[\begin{array}{ccc}
1 & 0 & \sqrt{2} \\
0 & 2 & 2 \\
\sqrt{2} & 2 & 4
\end{array}\right]
$$

should have eigenvalues of $\lambda_{1}(\widetilde{Q})=0, \lambda_{2}(\widetilde{Q})=1.4384$, $\lambda_{3}(\widetilde{Q})=5.5616$. The $\mathrm{C}$ bounds, SS bounds, and WS bounds for the eigenvalues of the matrix $\widetilde{Q}$ are listed in Table 1. For $\lambda_{1}(\widetilde{Q})$, the lower SS bound is best; next is the lower C bound followed by the lower WS bound; the upper SS bound is best; next is the upper WS bound followed by the upper $\mathrm{C}$ bound. For $\lambda_{2}(\widetilde{Q})$, SS bounds and C bounds are the same as the $\mathrm{C}$ bounds. For $\lambda_{3}(\widetilde{Q})$, the lower SS bound is best; the lower $\mathrm{C}$ bound and WS bound are the same; the upper SS bound is best; next is the upper WS bound followed by the upper C bound. In conclusion, the SS bounds are best.

The bounds of the eigengap of $Q$ provided by (69) are

$$
2<\mathrm{EG}_{1}(Q)<6
$$

while the bounds provided by (70) are

$$
2.6861<\mathrm{EG}_{1}(Q)<5.6458 .
$$

Therefore, the bounds by (70) are tighter than those by (69). This numerically justifies Proposition 19.

Example 21. We consider an arrowhead matrix $Q$ as follows:

$$
Q=\left[\begin{array}{ccc}
1 & 0 & \sqrt{2} \\
0 & 6 & 2 \\
\sqrt{2} & 2 & 7
\end{array}\right]
$$


TABLE 1: C bounds, SS bounds, and WS bounds for Example 20.

\begin{tabular}{|c|c|c|c|c|c|c|}
\hline \multirow{2}{*}{ Eigenvalues } & \multicolumn{2}{|c|}{ C bounds } & \multicolumn{2}{|c|}{ SS bounds } & \multicolumn{2}{|c|}{ WS bounds } \\
\hline & Lower & Upper & Lower & Upper & Lower & Upper \\
\hline$\lambda_{1}(\widetilde{Q})=0$ & -0.4142 & 1 & -0.3723 & 0.3542 & -1 & 0.6667 \\
\hline$\lambda_{2}(\widetilde{Q})=1.4384$ & 1 & 2 & 1 & 2 & & \\
\hline$\lambda_{3}(\widetilde{Q})=5.5616$ & 4 & 7.4142 & 5.3723 & 5.6458 & 4 & 5.6667 \\
\hline
\end{tabular}

TABLE 2: C bounds, SS bounds, and WS bounds for Example 21.

\begin{tabular}{|c|c|c|c|c|c|c|}
\hline \multirow{2}{*}{ Eigenvalues } & \multicolumn{2}{|c|}{ C bounds } & \multicolumn{2}{|c|}{ SS bounds } & \multicolumn{2}{|c|}{ WS bounds } \\
\hline & Lower & Upper & Lower & Upper & Lower & Upper \\
\hline$\lambda_{1}(Q)=0.6435$ & -0.4142 & 1 & 0.1270 & 1 & 0 & 2.3333 \\
\hline$\lambda_{2}(Q)=4.6304$ & 1 & 6 & 4 & 6 & & \\
\hline$\lambda_{3}(Q)=8.7261$ & 6 & 10.4142 & 7.8730 & 9 & 7 & 9.3333 \\
\hline
\end{tabular}

TABLE 3: C bounds, SS bounds, and WS bounds for Example 22.

\begin{tabular}{|c|c|c|c|c|c|c|}
\hline \multirow{2}{*}{ Eigenvalues } & \multicolumn{2}{|c|}{ C bounds } & \multicolumn{2}{|c|}{ SS bounds } & \multicolumn{2}{|c|}{ WS bounds } \\
\hline & Lower & Upper & Lower & Upper & Lower & Upper \\
\hline$\lambda_{1}(Q)=0.6192$ & 0.5000 & 1 & 0.2753 & 0.8820 & 0.2137 & 0.9402 \\
\hline$\lambda_{2}(Q)=1.3183$ & 1 & 2 & 1 & 2 & & \\
\hline$\lambda_{3}(Q)=3.0624$ & 2 & 3.5000 & 2.7247 & 3.1180 & 2.3931 & 3.1196 \\
\hline
\end{tabular}

The eigenvalues of $Q$ and the corresponding $C$ bounds, SS bounds, and WS bounds for the eigenvalues of $Q$ are listed in Table 2. For $\lambda_{1}(Q)$, the lower SS bound is the best; next is the lower WS bound followed by the lower C bound; the SS upper bound and the $\mathrm{C}$ upper bound are the same and are better than the upper WS bound. For $\lambda_{2}(Q)$, the lower SS bound is better than the lower $\mathrm{C}$ bound; the upper SS bound is the same as the upper $C$ bound. For $\lambda_{3}(Q)$, the lower SS bound is the best; next is the lower WS bound followed by the lower C bound; the upper SS bound is the best; next is the upper WS bound followed by the upper $\mathrm{C}$ bound.

Example 22. We consider an arrowhead matrix $Q$ as follows:

$$
Q=\left[\begin{array}{lll}
1 & 0 & \frac{1}{2} \\
0 & 2 & 1 \\
\frac{1}{2} & 1 & 2
\end{array}\right]
$$

The eigenvalues of $Q$ and the corresponding $C$ bounds, SS bounds, and WS bounds for the eigenvalues of $Q$ are listed in Table 3. For $\lambda_{1}(Q)$, the lower $C$ bound is best; next is the lower SS bound followed by the lower WS bound; the upper SS bound is the best; next is the upper WS bound followed by the upper $C$ bound. For $\lambda_{2}(Q)$, the SS bounds are the same as the C bounds. For $\lambda_{3}(Q)$, the upper SS bound is the best; next is the upper WS bound followed by the upper $\mathrm{C}$ bound; the SS lower bound is the best; next is the lower WS bound followed by the lower $\mathrm{C}$ bound.

\section{Conclusions}

Motivated by the need to more accurately estimate eigengaps of the system matrices associated with hub matrices, this paper provides an efficient way to estimate the lower and upper bounds of arrowhead matrices. Improved lower and upper bounds for the eigengaps of the system matrices are developed. We applied these results to a wireless computation application, and subsequently we presented several numerical examples. In the future, we will plan to extend our results to hub dominant matrices, which will allow hub matrices with correlated nonhub columns.

\section{Acknowledgments}

The research was supported by the Air Force Visiting Summer Faculty Program and by the US National Science Foundation under Grant DMS-0712827.

\section{References}

[1] M. Bixon and J. Jortner, "Intramolecular radiationless transitions," The Journal of Chemical Physics, vol. 48, no. 2, pp. 715726, 1968.

[2] J. W. Gadzuk, "Localized vibrational modes in Fermi liquids. General theory," Physical Review B, vol. 24, no. 4, pp. 1651$1663,1981$.

[3] D. P. O'Leary and G. W. Stewart, "Computing the eigenvalues and eigenvectors of symmetric arrowhead matrices," Journal of Computational Physics, vol. 90, no. 2, pp. 497-505, 1990.

[4] K. Dickson and T. Selee, "Eigenvectors of arrowhead matrices via the adjugate," preprint, 2007. 
[5] D. Boley and G. H. Golub, "A survey of matrix inverse eigenvalue problems," Inverse Problems, vol. 3, no. 4, pp. 595$622,1987$.

[6] B. Parlett and G. Strang, "Matrices with prescribed Ritz values," Linear Algebra and Its Applications, vol. 428, no. 7, pp. 1725-1739, 2008.

[7] J. Peng, X.-Y. Hu, and L. Zhang, "Two inverse eigenvalue problems for a special kind of matrices," Linear Algebra and Its Applications, vol. 416, no. 2-3, pp. 336-347, 2006.

[8] H. Pickmann, J. Egaña, and R. L. Soto, "Extremal inverse eigenvalue problem for bordered diagonal matrices," Linear Algebra and Its Applications, vol. 427, no. 2-3, pp. 256-271, 2007.

[9] H. T. Kung and B. W. Suter, "A Hub matrix theory and applications to wireless communications," EURASIP Journal on Advances in Signal Processing, vol. 2007, Article ID 13659, 8 pages, 2007.

[10] J. M. Kleinberg, "Authoritative sources in a hyperlinked environment," Journal of the ACM, vol. 46, no. 5, pp. 604-632, 1999.

[11] D. Tse and P. Viswanath, Fundamentals of Wireless Communication, Cambridge University Press, Cambridge, UK, 2005.

[12] R. A. Horn and C. R. Johnson, Matrix Analysis, Cambridge University Press, Cambridge, UK, 1985.

[13] E. Montano, M. Salas, and R. Soto, "Positive matrices with prescribed singular values," Proyecciones, vol. 27, no. 3, pp. 289-305, 2008.

[14] R. Bhatia, Matrix Analysis, vol. 169 of Graduate Texts in Mathematics, Springer, New York, NY, USA, 1997.

[15] H. Wolkowicz and G. P. H. Styan, "Bounds for eigenvalues using traces," Linear Algebra and Its Applications, vol. 29, pp. 471-506, 1980. 